\title{
Influence of pulse power amplitude on plasma properties and film deposition in high power pulsed plasma enhanced chemical vapor deposition
}

Daniel Lundin, Jens Jensen and Henrik Pedersen

\author{
Linköping University Post Print
}

\section{Tweet}

N.B.: When citing this work, cite the original article.

Original Publication:

Daniel Lundin, Jens Jensen and Henrik Pedersen, Influence of pulse power amplitude on plasma properties and film deposition in high power pulsed plasma enhanced chemical vapor deposition, 2014, Journal of Vacuum Science \& Technology. A. Vacuum, Surfaces, and Films, (32), 3.

http://dx.doi.org/10.1116/1.4867442

Copyright: American Vacuum Society http://www.avs.org/

Postprint available at: Linköping University Electronic Press

http://urn.kb.se/resolve?urn=urn:nbn:se:liu:diva-107122 


\title{
Influence of pulse power amplitude on plasma properties and film deposition in high power pulsed plasma enhanced chemical vapor deposition
}

\author{
Daniel Lundin \\ Laboratoire de Physique des Gaz et Plasmas, UMR 8578 CNRS, Université Paris Sud-XI, \\ F-91405 Orsay Cedex, France \\ Jens Jensen and Henrik Pedersen ${ }^{\text {a) }}$ \\ Department of Physics, Chemistry and Biology, Linköping University, SE-581 83 Linköping, Sweden
}

(Received 27 January 2014; accepted 21 February 2014; published 4 March 2014)

\begin{abstract}
The discharge characteristics in high power pulsed plasma enhanced chemical vapor deposition is studied with the aim to characterize the impact of high power pulses (HiPP). Using a power scheme of combined HiPP and direct current (DC) to ignite the plasma discharge, and adjusting the HiPP/DC time-averaged power ratio while keeping the total power constant, the effect of the high power pulses was isolated from the total power. By monitoring the discharge current along with the optical emission from the plasma, it is found that the amount of available ions increased with increasing HiPP/DC ratio, which indicates a higher plasma density. Using carbon films deposited from acetylene in an argon plasma as model system, a strong increase in deposition rate with higher HiPP/DC is observed. The increased deposition rate is ascribed to a more efficient plasma chemistry generated by the denser plasma. (C) 2014 American Vacuum Society.
\end{abstract}

[http://dx.doi.org/10.1116/1.4867442]

\section{INTRODUCTION}

In plasma enhanced chemical vapor deposition (PECVD), the energy stored in a plasma is used to excite, dissociate, and even ionize the precursor molecules for thin film synthesis, at a significantly lower overall temperature compared to thermally activated chemical vapor deposition. This enables thin film synthesis on temperature sensitive materials such as integrated circuit boards and plastics. The composition and charge state(s) of the deposition flux is governed by the plasma chemistry and is mainly mediated by inelastic collisions between electrons as well as excited and ionized gas atoms and the precursor molecules. ${ }^{1}$ The collisions in the plasma between, e.g., electrons and precursor molecules lead to excitation, dissociation, and ionization of the precursor molecules. To increase the rate of reactions to occur, such as

$$
\mathrm{e}^{-}+\mathrm{X}_{2} \rightarrow 2 \mathrm{X}+\mathrm{e}^{-}
$$

where $\mathrm{X}_{2}$ denotes a diatomic precursor molecule, the electron temperature and/or the electron density of the plasma must increase. A higher density of the electrons leads to an increased number of collisions, while an increased electron temperature results in a larger number of energetic electrons and thus generally a higher probability for the collision to lead to one of the desired reactions stated above. The electron density and temperature can be derived from the current-voltage characteristics of the plasma as measured by a Langmuir probe. $^{2}$ A typical electron density and electron temperature in PECVD is $10^{14}-10^{16} \mathrm{~m}^{-3}$ and $1 \mathrm{eV}$, respectively. ${ }^{1} \mathrm{~A}$ thin film deposition technique that results in electron densities on the order of $10^{18}-10^{19} \mathrm{~m}^{-3}$ is high power impulse magnetron sputtering (HiPIMS). The high plasma density in HiPIMS is

${ }^{\text {a)} E l e c t r o n i c ~ m a i l: ~ h e n k e @ i f m . l i u . s e ~}$ achieved by high-power pulses at a low duty factor $(<10 \%)$ and low frequency $(<10 \mathrm{kHz})$, leading to peak cathode power densities of the order of several $\mathrm{kW} \mathrm{cm}^{-2}{ }^{3}$ A PECVD technique using a HiPIMS-like power scheme, denoted high power pulsed PECVD (HiPP-PECVD), has recently been outlined. ${ }^{4}$ This HiPP-PECVD technique is the focus of the present work.

Albeit no direct measurements of the electron density in HiPP-PECVD have so far been possible due to the process pressure of $65-175 \mathrm{~Pa}^{4}$ which is out of range for the collisionless probe theory used for the required Langmuir probes measurements, ${ }^{5}$ several encouraging results from early HiPP-PECVD have been reported: During deposition of amorphous carbon films, a larger fraction of ionized species has been detected in the plasma by optical emission spectroscopy (OES) when high power pulses (HiPP) are used compared to using only direct current (DC) plasma discharges. ${ }^{6}$ Also a higher deposition rate for the same flow of precursor gas and the same time averaged power when using a combination of $\mathrm{HiPP}+\mathrm{DC}$ compared to DC power has been observed. ${ }^{6}$ In combination, these observations point toward an increased dissociation of the precursor gas molecules likely due to an increased plasma density for HiPP-PECVD as compared to DC-driven PECVD. In addition, during deposition of alumina films a lower deposition temperature for $\alpha$-alumina was allowed by the use of HiPP-PECVD. ${ }^{7}$ The lower deposition temperature was ascribed to the more efficient plasma and surface chemistry in the HiPP-PECVD reactor, leading to better cracking of the $\mathrm{AlCl}_{3}$ precursors and thereby less chlorine incorporated in the films.

Since one can tune not only the power delivered in each pulse but also the pulse length and the pulse frequency, HiPP-PECVD could offer new ways of controlling the plasma chemistry and thereby the film deposition in PECVD. For this, a fundamental understanding of how the plasma chemistry and thereby the deposition process can be 
controlled by the plasma discharge is first needed. A step towards such an understanding is the finding that by pulsing the plasma in "standard" PECVD, i.e., by switching it on and off, and carefully selecting the precursor chemistry, selflimiting growth, similar to atomic layer deposition, of metal oxides $^{8-11}$ and recently also sulphides, ${ }^{12}$ can be achieved without pulsing the precursor gases. The pulsed nature of HiPP-PECVD could potentially further advance this type of PECVD. It is thus of great importance for successful use of HiPP-PECVD to fully understand how the plasma and film deposition are affected by the discharge characteristics.

In this work, we have used carbon films deposited from acetylene in an argon plasma as model system and investigated the effects of the HiPP discharge current pulse amplitude by superposition of HiPP and DC discharges with varied HiPP/DC time-averaged power ratio. Keeping the total average power constant, the plasma discharge as well as the thin film deposition rate and thin film composition were studied. Focus is on correlating changes in the deposition rate and thin film composition with changes in the plasma composition.

\section{EXPERIMENTAL DETAILS}

The stainless steel HiPP-PECVD reactor used in this study has previously been described in detail. ${ }^{6}$ In the reactor, the plasma is generated by a hollow cathode discharge in a copper cathode placed in the top lid of the reaction chamber over the substrate. The cathode-substrate distance was $30 \mathrm{~mm}$. The diameter and length of the hollow cathode was 5 and $54 \mathrm{~mm}$, respectively, which gives an inner cathode area of $850 \mathrm{~mm}^{2}$. Argon gas (minimum purity of 99.9997\%) controlled by a mass flow controller, was fed through the hollow cathode enabling a brightly glowing argon plasma jet extending approximately $10 \mathrm{~mm}$ from the hollow cathode towards the substrate. The argon flow was set at $84 \mathrm{sccm}$ in all experiments. Carbon film deposition was done by feeding acetylene $\left(\mathrm{C}_{2} \mathrm{H}_{2}\right)(99.6 \%)$ into the plasma jet via a $65 \mathrm{~mm}$ diameter gas ring surrounding the orifice of the hollow cathode. The acetylene flow was controlled by a mass flow controller to $20 \mathrm{sccm}$ in all experiments. All experiments were performed in flow controlled mode, i.e., without throttle valve to control the total pressure; the process pressure was $80 \mathrm{~Pa}$ throughout this study.

The plasma was ignited by a combination of HiPP and DC for more stable operating conditions, such as easy ignition and reduced arcing. The same setup has been shown to be beneficial also for HiPIMS. ${ }^{13}$ A DC power supply (MDX 500, Advanced Energy) and a high-power pulsed power supply ( $\mathrm{HiP}^{3}$, Ionautics) were connected in parallel to the cathode. The DC power supply was isolated from the HiPP power supply by a series diode between DC supply and cathode. For pure DC processes, only the DC power supply was connected to the hollow cathode. The high power pulses were $30 \mu$ s long, delivered with a frequency of $500 \mathrm{~Hz}$, giving an overall duty factor of $1.5 \%$. Discharge parameters, such as cathode voltage, $U_{D}$, and cathode current, $I_{D}$, were monitored and recorded on a Tektronix TDS2004B oscilloscope. A spectrometer (Mechelle Sensicam 900) connected to a collimator via an optical fiber, was used to record time-averaged optical emission from the plasma in the spectral range of 300-1100 nm.

Single crystalline (100) silicon wafers, cut into approximately $20 \times 20 \mathrm{~mm}^{2}$ pieces, ultrasonically cleaned in ethanol for $10 \mathrm{~min}$ and blow dried in dry nitrogen before loading, were used as substrates for film deposition. During deposition, the ions in the plasma were attracted towards the substrate surface using a negative bias of $-80 \mathrm{~V} \mathrm{DC}$. The anode was places below the substrate to further guide the plasma towards the substrate, an anode bias of $+40 \mathrm{~V}$ DC was used throughout this study. The substrate temperature was measured by a thermocouple (type K) mounted on the back side of the $30 \times 30 \times 2 \mathrm{~mm}^{3}$ steel sample holder. The thermocouple was connected for temperature measurements immediately after plasma shut off after deposition to avoid any false signals in the thermocouple due to the plasma currents. Film thickness and microstructure were studied using a LEO scanning electron microscope (SEM) on cleaved samples. Time of flight-energy elastic recoil detection analysis (ToF-E ERDA) was used to determine the elemental composition of the films using $36 \mathrm{MeV}$ iodine ions. The experimental details of the ToF-E ERDA can be found elsewhere. ${ }^{14,15}$

\section{RESULTS AND DISCUSSION}

A series of experiments were made, where we systematically increased the portion of the power to the plasma delivered in the form of HiPP by increasing the discharge current and voltage during the pulse. The experiments ranged from pure DC power, i.e., a HiPP/DC ratio of 0 and going up to a HiPP/DC ratio $>1$, i.e., more than half of the power was delivered as HiPP. It should be pointed out that the plasma discharges were monitored in real time during deposition and the HiPP power was calculated as time-averaged power. In all experiments, the total time-averaged power, $\left\langle\mathrm{P}_{\text {tot }}\right\rangle$ $=\left\langle\mathrm{P}_{\mathrm{HiPP}}\right\rangle+\mathrm{P}_{\mathrm{DC}}$, was adjusted to give $\left\langle\mathrm{P}_{\text {tot }}\right\rangle=100 \pm 10 \mathrm{~W}$.

As mentioned in the Introduction, the plasma (electron) density has so far not been directly measured by conventional Langmuir probes in this HiPP-PECVD setup due to the high process pressure. Thus, the plasma density must be estimated by other means. Since plasmas are considered to be quasi-neutral, the ion density is proportional to the electron density and by studying the amount of ions in the plasma; one can indirectly study the number of electrons in the plasma. In Fig. 1, discharge current curves recorded during process with different HiPP/DC ratios are given. For the same time-averaged power, the peak discharge current shows a significant increase with increasing HiPP/DC. Similar curves have been reported for HiPIMS, showing a direct correlation between the discharge current and the electron (plasma) density. ${ }^{16,17}$ The increase in discharge current with higher HiPP/DC ratio in Fig. 1 resulting in a larger amount of charge carriers can thus be interpreted as an increase in plasma density with increasing HiPP/DC ratio. It should, however, be pointed out that it is only a relative increase in the plasma density that can be extracted from 


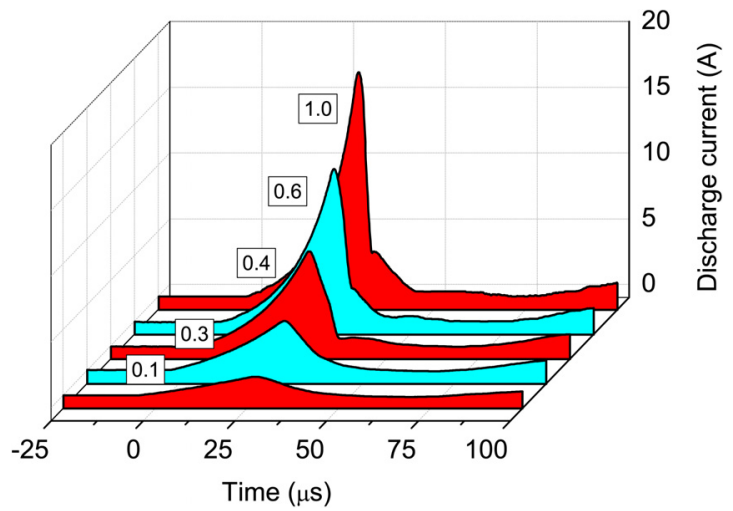

FIG. 1. (Color online) Recorded discharge currents during the HiPP pulse for increasing HiPP/DC ratios. The inserted captions give the HiPP/DC ratio associated with each current plot.

Fig. 1, and that no absolute values of the plasma density can be extracted from these discharge curves.

The plasma composition was therefore investigated in more detail using OES for the different HiPP/DC ratios. It was possible to identify changes in the degree of ionization between of the elements of interest, $\mathrm{C}$ and $\mathrm{Ar}$ from process gases and $\mathrm{Cu}$ from sputtering of the hollow cathode, for the different discharge configurations. These measurements are an extension to what we have reported earlier. ${ }^{6}$ Details of specific emission lines, as well as typical OES spectra, can be found in that publication and will not be repeated here. Striking qualitative differences between the pure DC-driven and the superimposed HiPP/DC discharges are: The DCdriven discharge $(\mathrm{HiPP} / \mathrm{DC}=0)$ shows large fractions of neutral copper $(\mathrm{Cu} \mathrm{I})$, whereas the emission from the discharges with higher HiPP/DC also shows singly ionized copper (Cu II). Furthermore, emission of singly ionized argon (Ar II) and singly ionized carbon (C II) are only found for HiPP/DC $>0$. No emission from neutral hydrogen (H I) nor ionized hydrogen was detected, which is in line with our previous study. ${ }^{6}$

Quantitative trends could not be established due to the weak signal of the ion lines compared to strong emission from neutral argon along with the fact that any significant changes during the discharge pulse were likely dampened due to the nature of the time-averaged OES measurements. Due to the low signal-to-noise ratio, we can therefore not establish with good enough accuracy a possible intensity increase with increasing HiPP/DC ratio, although an increase going from $\mathrm{HiPP} / \mathrm{DC}=0$ to $\mathrm{HiPP} / \mathrm{DC}>0$ was clearly detected. This indicates that by superimposing the HiPP signal on the DC signal, the ionized flux fraction of the depositing species increases.

When using the HiPP-PECVD setup to deposit carbon films, the deposition rate increases with increasing HiPP/DC ratio (Fig. 2), i.e., more film is deposited from the same amount of precursor gas at the same total time-averaged power by using a higher HiPP/DC ratio. Based on the discharge current characteristics (Fig. 1) and the OES results, we propose that the increased deposition rate is due to the higher degree of ionization in the plasma at higher HiPP/DC

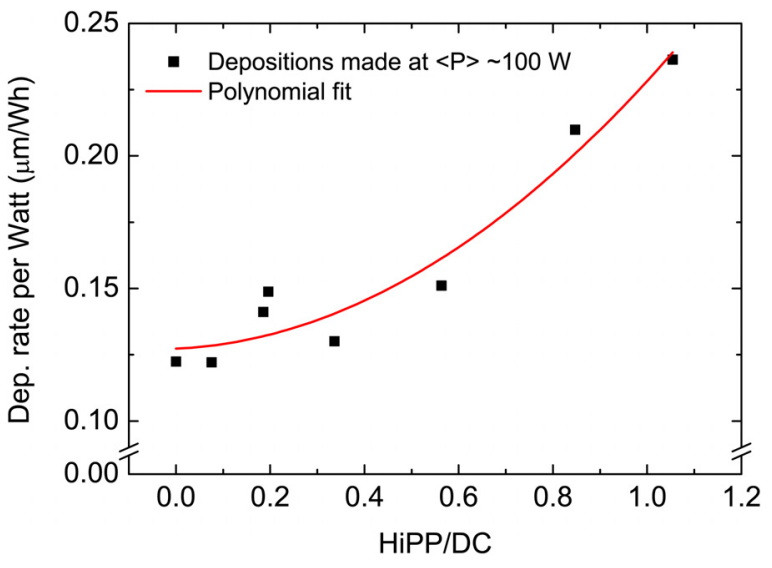

FIG. 2. (Color online) Deposition rate of carbon films for different HiPP/DC ratios. Note that the deposition rate is plotted as $\mu \mathrm{m} / \mathrm{h}$ per watt of power supplied to the cathode to account for slight deviations in the total power supplied to the cathode. The time averaged power were $100 \pm 10 \mathrm{~W}$ in all experiments.

ratios, which leads to more frequent dissociative electron/ion-acetylene collisions per time unit, which increases the number of reactive $\mathrm{C}$ species, and thus a better use of the precursor molecules. The deposited films all appear dense with a columnar microstructure, as shown in Fig. 3. No changes in the film microstructure with different HiPP/DC ratios could be observed from SEM; hence, the increased deposition rate is likely not a result of reduced film density. Furthermore, studies on HiPIMS discharges have shown film densification by operating in the HiPP mode as compared to DC mode ${ }^{18}$ which would effectively decrease the deposition rate measured using SEM.

It was previously shown that carbon films deposited with the current HiPP-PECVD setup contained significant amounts of copper, 30-50 at. \%, emanating from sputtering of copper from the hollow cathode. ${ }^{6}$ One could thus suspect that the increase in deposition rate with higher HiPP/DC is due to an increased sputtering of copper. However, film composition measurements by ToF-E ERDA show the opposite trend: the film deposited at $\mathrm{HiPP} / \mathrm{DC}=0$, i.e., pure $\mathrm{DC}$ power, contains 65 at. \% copper (29 at. \% C), while using $\mathrm{HiPP} / \mathrm{DC}=0.56$, the copper content decreases to 56 at. $\%$

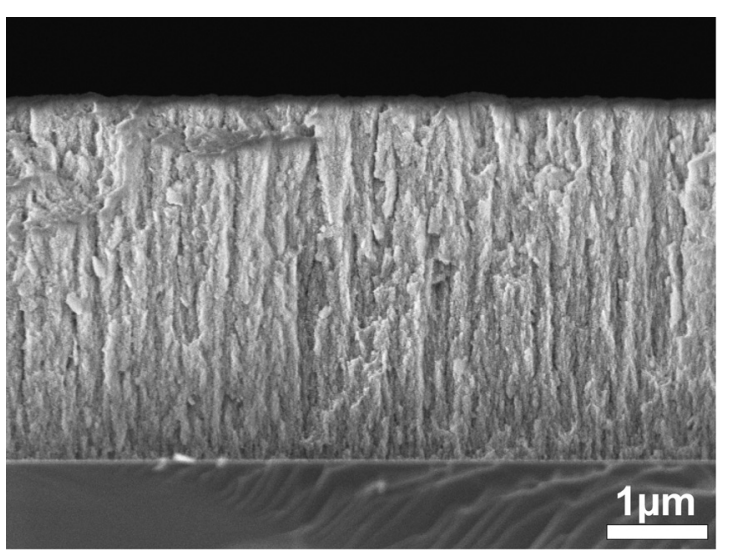

FIG. 3. Cross section SEM micrograph of a $3.6 \mu \mathrm{m}$ thick carbon-copper film deposited in $10 \mathrm{~min}$ with $\mathrm{HiPP} / \mathrm{DC}=0.85$. 
(37 at. \% C). Finally, the film deposited at HiPP/DC $=1.05$ shows the lowest copper content; 44 at. \% (46 at. \% C). In our previous study, it was found that the copper content of the a-C films decreased with higher deposition rate and when the films were deposited using HiPP + DC power compared to DC power, which is in line with the present results. ${ }^{6}$ One reason for the reduced amount of $\mathrm{Cu}$ in the coating with increasing HiPP/DC fraction is likely to be the ionization of sputtered neutral $\mathrm{Cu}$ in the close vicinity of the hollow cathode (i.e., the densest plasma region) followed by backattraction of the positively charged ion to the negatively charged cathode surface. This is a well-known effect in magnetron sputtering operating in the HiPP mode (HiPIMS), which reduces the deposition rate at the substrate. ${ }^{19}$ In HiPIMS modeling using similar pulse power densities, the reduction of material flux to the substrate due to backattraction has been estimated to $\sim 60 \%$ during the peak of the pulse. ${ }^{20}$ In addition, the sputter contribution to the deposition rate has, in the case of chromium deposition by HiPIMS, been shown to decrease with higher HiPP/DC ratio; the deposition rate in pure HiPIMS was only $60 \%$ of the deposition rate under pure DC conditions. ${ }^{13}$ Further analysis of the HiPP-PECVD plasma is needed in order to determine this reduction more precisely. However, we can still conclude that the increased deposition rate with higher HiPP/DC power rate is not due to an increased sputtering of copper but rather to a more efficient use of the acetylene precursor.

From ToF-E ERDA, it was also found that the hydrogen content is $4-8$ at. \% in the films, corresponding to a $\mathrm{H} / \mathrm{C}$ of $0.15 \pm 0.02$. The substrate temperature, as measured on the back side of the steel sample holder just after deposition was $63 \pm 6{ }^{\circ} \mathrm{C}$ without any dependence on HiPP/DC. This temperature measurement is a crude way of estimating the temperature at the substrate. We have here assumed that the temperature on the substrate side of the sample holder is similar to the back side, where the temperature is measured given the thermal conductivity of steel. The temperature on the silicon substrate surface may differ by a few tens of degrees, but the substrate temperature can still be considered fairly low. The low substrate temperature means that the low hydrogen content of the films should be attributed to plasma chemical breakdown of acetylene and/or surface chemical reactions mediated by the ion bombardment. The concentrations of other impurities were very low; oxygen and nitrogen levels of around 1 at. \% were detected; no argon was detected in the films.

Worth mentioning is that an increase of the pulse discharge current amplitude (or similarly pulse power amplitude) is not the only way to modify the HiPP/DC ratio. It is also possible to modify the pulse width as well as the pulse frequency (duty factor). Changing the pulse frequency was in the present case not motivated, since an increase in the frequency $(>500 \mathrm{~Hz})$ while keeping the other discharge parameters constant would lead to exceeding the maximum time-averaged power that can be tolerated without damaging and/or melting the cathode. Decreasing the pulse frequency would allow even higher instantaneous discharge currents than investigated in the present work, but such tests have so far resulted in unstable operation (arc discharges and secondary discharges between the hollow cathode isolation and the chamber walls).

Similarly, increased pulse width, $>30 \mu \mathrm{s}$, (while staying below the maximum time-averaged power) also led to an unstable discharge, while shorter pulse widths resulted in insufficient time for the initial current rise to reach high current values. It was therefore concluded that the chosen pulse conditions were a good balance in the present HiPP-PECVD system. However, future investigations using for example other material systems will most likely require a reoptimization of the parameters above.

\section{SUMMARY AND CONCLUSIONS}

When increasing the HiPP/DC ratio, i.e., fraction of the power delivered to the discharge in the form of high power pulses, a strong increase of the deposition rate is noticed for the same flow of precursor molecules and the same amount of time-averaged power. This increase in deposition rate is attributed to a more efficient use of the precursor molecules. Discharge currents recorded for plasma discharges with increasing HiPP/DC ratio in the delivered power combined with plasma chemistry studies by optical emission spectroscopy indicates that the plasma density increases with increasing HiPP/DC ratio for the same amount of timeaveraged power. Despite that no quantitative determination of the increase in ion fraction could be established, the results in this study indicates that the plasma density in HiPP-PECVD can be increased by increasing the HiPP/DC ratio in the discharge power. The increased plasma density is suggested to lead to more efficient plasma chemistry where a higher amount of the precursor molecules are utilized for the thin film deposition.

\section{ACKNOWLEDGMENTS}

Financial support from the Swedish innovation agency (VINNOVA) and Ångpanneföreningens forskningsstiftelse (ÅForsk) is gratefully acknowledged. Ionautics AB is gratefully acknowledged for providing a $\mathrm{HiP}^{3}$ power supply. D.L. also gratefully acknowledges the financial support provided by the Swedish Research Council (VR) through his postdoctoral fellowship.

${ }^{1}$ W. Hess and D. B. Graves, in Chemical Vapor Deposition: Principles and Applications, edited by M. L. Hitchman and K. F. Jensen (Academic, San Diego, 1993), pp. 385-435.

${ }^{2}$ H. Mott-Smith and I. Langmuir, Phys. Rev. 28, 727 (1926).

${ }^{3}$ D. Lundin and K. Sarakinos, J. Mater. Res. 27, 780 (2012).

${ }^{4}$ D. Lundin and H. Pedersen, Phys. Procedia 46, 3 (2013).

${ }^{5}$ V. I. Demidov, S. V. Ratynskaia, and K. Rypdal, Rev. Sci. Instrum. 73, 3409 (2002)

${ }^{6}$ H. Pedersen, P. Larsson, A. Aijaz, J. Jensen, and D. Lundin, Surf. Coat. Technol. 206, 4562 (2012).

${ }^{7}$ K. Jiang, K. Sarakinos, S. Konstantinidinis, and J. M. Schneider, J. Phys. D 43, 325202 (2010).

${ }^{8}$ M. Seman, J. J. Robbins, S. Agarwal, and C. A. Wolden, Appl. Phys. Lett. 90, 131504 (2007).

${ }^{9}$ M. T. Seman, D. N. Richards, P. Rowlette, and C. A. Wolden, Chem. Vap. Deposition 14, 296 (2008).

${ }^{10}$ P. C. Rowlette, C. G. Allen, O. B. Bromley, and C. A. Wolden, J. Vac. Sci. Technol. A 27, 761 (2009). 
${ }^{11}$ P. C. Rowlette and C. A. Wolden, ACS Appl. Mater. Interfaces 1, 2586 (2009).

${ }^{12}$ C. D. Sentman, M. O'Brien, and C. A. Wolden, J. Vac. Sci. Technol. A 32, 021201 (2014).

${ }^{13}$ M. Samuelsson, D. Lundin, K. Sarakinos, F. Björefors, B. Wälivaara, H. Ljungcrantz, and U. Helmersson, J. Vac. Sci. Technol. A 30, 031507 (2012).

${ }^{14}$ J. Jensen, D. Martin, A. Surpi, and T. Kubart, Nucl. Instrum. Methods Phys. Res. B 268, 1893 (2010).

${ }^{15}$ H. J. Whitlow, G. Possnert, and C. S. Petersson, Nucl. Instrum. Methods Phys. Res. B 27, 448 (1987).
${ }^{16}$ J. Alami, J. T. Gudmundsson, J. Bohlmark, J. Birch, and U. Helmersson, Plasma Sources Sci. Technol. 14, 525 (2005).

${ }^{17} \mathrm{C}$. Huo, "Modeling and experimental studies of high power impulse magnetron sputtering discharges," Doctoral thesis (KTH Royal Institute of Technology, Stockholm, 2013).

${ }^{18}$ M. Samuelsson, D. Lundin, J. Jensen, M. A. Raadu, J. T. Gudmundsson, and U. Helmersson, Surf. Coat. Technol. 205, 591 (2010).

${ }^{19}$ D. J. Christie, J. Vac. Sci. Technol. A 23, 330 (2005).

${ }^{20}$ C. Huo, M. A. Raadu, D. Lundin, J. T. Gudmundsson, A. Anders, and N. Brenning, Plasma Sources Sci. Technol. 21, 045004 (2012). 